\title{
Daptomycin is not active against rapidly growing mycobacteria
}

Rapidly growing mycobacteria, formerly referred as to 'Group IV' in the Runyon classification, are being increasingly described as causative agents of infectious diseases, mainly respiratory diseases in cystic fibrosis patients (Mycobacterium abscessus) and either community-acquired (Mycobacterium fortuitum) or health-careassociated (Mycobacterium chelonae) cutaneous infections. The number of antibiotics available to treat infections caused by these organisms is very limited and most of them are poorly active.

Treatment usually relies on antibiotic combinations given intravenously for several months. Consequently, new drugs are urgently needed to treat infections due to rapidly growing mycobacteria. Recently, tigecycline and to a less extent linezolid have been shown to have some in vitro activity against rapidly growing mycobacteria (Brown-Elliott et al., 2003; Martin-de-Hijas et al., 2008).

Daptomycin, the first in a class of agents known as lipopeptides, is a novel antimicrobial agent used for the treatment of Gram-positive infections (Hawkey, 2008). The compound has a distinctive mechanism of action in that it exerts its bactericidal activity by disrupting plasma membrane function without penetrating into the cytoplasm. Daptomycin is active against multidrug-resistant, Gram-positive bacteria such as meticillin-resistant Staphylococcus aureus, vancomycin- resistant enterococci, and glycopeptideintermediate and -resistant $S$. aureus. To our knowledge, daptomycin has not been evaluated against mycobacteria.

We prospectively evaluated the in vitro activity of daptomycin against rapidly growing mycobacteria received at the National Reference Center for Mycobacteria (NRC) for susceptibility testing. We included in the study 40 strains involved in pulmonary $(n=32)$, skin and soft tissue $(n=5)$ or other $(n=3)$ infection. All the strains were identified by sequencing the heat-shock protein 65 gene. Mycobacteria were identified as $M$. abscessus (22), M. chelonae (7), $M$. fortuitum (5), Mycobacterium massiliense (4) and Mycobacterium bolletii (2). The MIC of daptomycin was measured by Etest on Mueller-Hinton medium. Readings were taken after 3-7 days of incubation at $30{ }^{\circ} \mathrm{C}$ depending on growth of the organism.

MICs are presented in Table 1.

Daptomycin MICs were $>64 \mathrm{mg} \mathrm{l}^{-1}$ for 33 of the 40 mycobacteria tested. The MIC was $>256 \mathrm{mg} \mathrm{l}^{-1}$ for M. chelonae, $M$. massiliense and $M$. bolletii. MICs were lower $\left(48 \mathrm{mg} \mathrm{l}^{-1}\right)$ against 3 of the 22 strains of $M$. abscessus, and M. fortuitum showed the most sensitive pattern with the MIC being $\leqslant 64 \mathrm{mg} \mathrm{l}^{-1}$.

According to the European Committee on Antimicrobial Susceptibility Testing, the

Table 1. MIC of daptomycin against clinical strains of $M$. abscessus, M. massiliense, $M$. chelonae, $M$. bolletii and $M$. fortuitum received at the NRC

\begin{tabular}{|c|c|c|c|c|c|c|c|c|}
\hline & \multicolumn{7}{|c|}{$\operatorname{MIC}\left(\mathrm{mg} \mathrm{l}^{-1}\right)$} & \multirow[b]{2}{*}{ Total } \\
\hline & 32 & 48 & 64 & 96 & 128 & 256 & $>256$ & \\
\hline M. abscessus & & 3 & & & 1 & 1 & 17 & 22 \\
\hline M. massiliense & & & & 1 & & & 3 & 4 \\
\hline M. chelonae & & & & & & & 7 & 7 \\
\hline M. bolletii & & & & & & & 2 & 2 \\
\hline M. fortuitum & 1 & 1 & 2 & & & & 1 & 5 \\
\hline Total & 1 & 4 & 2 & 1 & 1 & 1 & 30 & 40 \\
\hline
\end{tabular}

MICs of daptomycin against staphylococci and streptococci considered susceptible to this drug were below $1 \mathrm{mg} \mathrm{l}^{-1}$ (EUCAST, 2008). Taking into account this breakpoint, daptomycin should be considered as inactive against rapidly growing mycobacteria. However, considering that an MIC below the peak serum level of the drug can be predictive of some in vivo activity, daptomycin could have some activity in vivo. Indeed, the peak serum level generated by the usual dosing $4 \mathrm{mg} \mathrm{kg}^{-1}$ ranges from 57 to $77 \mathrm{mg} \mathrm{l}^{-1}$ (Hawkey, 2008). Thus, according to the MIC measured in this experiment, daptomycin could have some activity against a small fraction of the strains of $M$. abscessus and against the species $M$. fortuitum, which is the most sensitive of the five tested species. However, this activity is likely to be weak.

Even considering that MICs measured by E-test are often a little higher than those measured by the microdilution method, daptomycin will not be an antibiotic of first choice for treating infection due to rapidly growing mycobacteria (Woods et al., 2000).

\section{Sylvaine Bastian, ${ }^{1,3}$ Florence Brossier, 1,2,3 Claudine Wichlacz, ${ }^{1,3}$ Vincent Jarlier ${ }^{1,2,3}$ and Nicolas Veziris ${ }^{1,2,3}$}

${ }^{1}$ AP-HP, Hôpital Pitié-Salpêtrière, Laboratoire de Bactériologie-Hygiène, F-75013 Paris, France

${ }^{2}$ UPMC Université Paris 06, EA 1541, Laboratoire de Bactériologie-Hygiène, F-75005, Paris, France

${ }^{3}$ Centre National de Référence des Mycobactéries et de la Résistance des Mycobactéries aux Antituberculeux, F-75013 Paris, France

\section{Correspondence: Nicolas Veziris (nicolas.veziris@upmc.fr)}

Brown-Elliott, B. A., Crist, C. J., Mann, L. B., Wilson, R. W. \& Wallace, R. J., Jr (2003). In vitro 
activity of linezolid against slowly growing nontuberculous mycobacteria. Antimicrob Agents Chemother 47, 1736-1738.

EUCAST (2008). Miscellaneous antimicrobials EUCAST clinical MIC breakpoints 2008-06-19 (v 2.2). Retrieved 25/06/09, from http:// www.srga.org/eucastwt/MICTAB/ MICmiscellaneous.html
Hawkey, P. M. (2008). Pre-clinical experience with daptomycin. J Antimicrob Chemother 62 (Suppl. 3), iii7-iiil4.

Martin-de-Hijas, N. Z., Fernandez-Roblas, R., Fernandez-Martínez, A. I., Gadea, I. \&

Esteban, J. (2008). Usefulness of the

Etest for studying tigecycline susceptibility

of non-pigmented rapidly growing mycobacteria. Int J Antimicrob Agents 32, 366-367.

Woods, G. L., Bergmann, J. S., Witebsky, F. G., Fahle, G. A., Boulet, B., Plaunt, M., Brown, B. A., Wallace, R. J., Jr \& Wanger, A. (2000). Multisite reproducibility of Etest for susceptibility testing of Mycobacterium abscessus, Mycobacterium chelonae, and Mycobacterium fortuitum. J Clin Microbiol 38, 656-661. 